\title{
NOTE ON THE EQUIVALENCE OF CERTAIN PROPERTIES OF ABSTRACT SETS*
}

BY R. G. PUTNAM

Sierpinski $\nmid$ has shown that, in classes $(S)$ (that is, classes $(L)$ in which every derived set is closed), the following three properties are equivalent:

(1) Every uncountable set contains at least one point of condensation.

(2) Every clairsemé set is at most countable.

(3) Every infinite well ordered descending sequence of closed distinct sets is countable.

The equivalence of these three properties is shown by proving that property (1) implies (2), that (2) implies (3) and that (3) implies (1). The condition that every derived set be closed is used only in showing that (3) implies (1).

Sierpinski also proves in the same paper that, in classes $(S)$, properties (4) and (5) which follow are equivalent:

(4) Every infinite well ordered ascending sequence of closed distinct sets is countable.

(5) Every set $E$ of the class contains a countable subset $D$ dense in $E$ (that is, $E \subset D+D^{\prime}$ ).

The equivalence of these two properties is obtained by proving that (4) implies (5) and that (5) implies (4). The condition that every derived set be closed is used only in the proof that (4) implies (5).

Properties (3) and (4) are shown by means of examples to be independent of each other.

It is the purpose of this note to point out that the above equivalences hold without change for classes $(V)$ in which every derived set is closed and from this result theorems concerning classes $(V)$ are obtained. The method of showing the equivalences in classes $(V)$ is the same as that used for classes $(L)$, definitions of limit point, point of condensation, etc., in classes

* Presented to the Society, December 29, 1929.

$\dagger$ Sur l'équivalence de trois propriétés des ensembles abstraits, Fundamenta Mathematicae, vol. 2, p. 179. 
$(L)$ being replaced by those which apply to classes $(V)$. For example, in classes $(L)$ a point of condensation of a set $E$ is a limit point of $E$ which remains a limit point of every set obtained by removing a countable set of points from $E$; in classes $(V)$ a point of condensation of a set $E$ is a point in every neighborhood of which there is an uncountable set of points of $E$.

From the proof that property (1) implies property (2) we have, for all classes $(V)$, the following theorem.

THEOREM. In a class $(V)$ condensed in itself, ${ }^{*}$ every uncountable set $E$ is the sum of two disjoined sets, one of which is dense in itself (and not empty) and the other clairsemé and at most countable.

Since property (1) implies property (2) which in turn implies Property (3) in any class $(V)$, we may state the theorem:

THEOREM. In every class $(V)$ condensed in itself, every infinite descending sequence of distinct closed sets is countable.

This theorem has been shown by Fréchet $\dagger$ to hold for perfectly separable sets. $\ddagger$

From the fact that property 5 implies property 4 we obtain the following theorem.

THEOREM. Every infinite well ordered ascending sequence of closed distinct sets of a separable class $(V)$ is countable.

New York University

* A set is condensed in itself if every uncountable subset contains at least one point of condensation.

$\dagger$ Fundamenta Mathematicae, vol. 10, p. 334.

$¥$ A set is perfectly separable if its limit points can be defined in terms of a countable family of neighborhoods. A perfectly separable set is condensed in itself. See Fréchet, Les Espaces Abstraits, p. 233. 